\title{
Anaerobic Strength and Power in Master Athletes
}

\section{(ㄷ) (2) (1) (5)}

Author

Jelena Z Popadic Gacesa

\section{Affiliation}

Laboratory for functional diagnostics, Department of Physiology, Faculty of Medicine, University of Novi Sad, Faculty of Sport and Physical Education, University of Novi Sad

Key words

anaerobic power, strength, WAnT, ergometry, master athletes, ageing

received 06.10 .2016

revised 29.11.2016

accepted $\quad 01.12 .2016$

\section{Bibliography}

DOI http://dx.doi.org/10.1055/s-0042-123695

Published online: 2017 | Sports Medicine International Open

2017; 1: E30-E36

(c) Georg Thieme Verlag KG Stuttgart · New York

ISSN 2367-1890

Correspondence

Jelena Z Popadic Gacesa, MD, PhD

Faculty of Medicine

Department of Physiology

University of Novi Sad

3, Hajduk-Veljko St

21000 Novi Sad

Serbia

Tel.: + 381/21/6624 163, Fax: + 381/21/6624 163

jpopadic@uns.ac.rs

jelena.popadic-gacesa@mf.uns.ac.rs

\section{ABSTRACT}

The study compared anaerobic capacity and power in master athletes of different ages, and correlated 2 different anaerobic tests. The study was conducted on 60 male athletes: group I $30-35$ years $\left(n_{1}=35\right.$, age $32.23 \pm 1.53 \mathrm{y})$, group $\| 35-45$ years $\left(n_{2}=15\right.$, age $\left.39.17 \pm 3.60 y\right)$, group III above 46 years $\left(n_{3}=10\right.$, age $\left.52.13 \pm 4.50 y\right)$. The Wingate anaerobic test and ergometry of elbow flexors, elbow extensors, and knee extensors were performed. Average peak anaerobic power (PP) in group I was $692.94 \pm 216.53 \mathrm{~W}$, in group II 593.40 $\pm 185.91 \mathrm{~W}$, non-significantly lower by $14.4 \%(p>0.05)$. In group III, PP was $381.50 \pm 117.62 \mathrm{~W}$, significantly lower by $44.9 \%$ when compared with group I ( $\leq \leq 0.001)$. Ergometry parameter of muscle strength and power showed greater decline with age (up to $32.3 \%$ ) in comparison with contraction velocity (up to $14.1 \%$ ). Significant correlation was registered between PP and ergometric parameter power for knee extensors (KE) (PP/KE power: $r$ was 0.59 in group I, 0.85 in group II, and 0.90 in group III, $p \leq 0.05$ ). Master athletes represent an interesting population for studying the chronic effects of physical activity on functional capacity changes during ageing. Strength parameters "age" in different fashion, because the contraction velocity parameter is less influenced than strength by the muscle alterations with increasing age.

$\begin{array}{ll}\text { Abbreviations } \\ \text { BH } & \text { body height } \\ \text { BW } & \text { body weight } \\ \text { Cl } & \text { confidence interval } \\ \text { EE } & \text { elbow extensors } \\ \text { EF } & \text { elbow flexors } \\ \text { EP } & \text { explosive power } \\ \text { FI } & \text { fatigue index } \\ \text { KE } & \text { knee extensors } \\ \text { L } & \text { load } \\ \text { Lmax } & \text { maximal load } \\ \text { MP } & \text { mean power } \\ \text { MS } & \text { muscle strength } \\ \text { MSmax } & \text { maximal muscle strength } \\ \text { P } & \text { power } \\ \text { P30 } & \text { power output in } 30^{\text {th }} \text { second } \\ \text { PD } & \text { power decline } \\ \text { PP } & \text { peak power } \\ \text { SD } & \text { standard deviation }\end{array}$

\section{Vel contraction velocity \\ W work \\ WAnT Wingate anaerobic test}

\section{Introduction}

Master athletes present an ageing athletic population which is engaged in regular training programmes and participation in competitions. They are an ideal model for investigating physiological changes related to the ageing process. The population of master athletes is growing as more athletes remain active at the competition level in their sports far after 30 years of age, which allows experts to study elite performance and its decline after the third decade of life, with all its variations in different sports and training regimens, and to compare it to performance in older populations with lower levels of physical activity.

The effect of programmed training increases functional capacity in order to improve a person's physical performance and health. In athletes, maximal values of functional capacity are achieved several years after starting the training, chronic effects are maintained 
for a long time and in most sports plateau at 25-30 years of athletes' age. Then a gradual linear decline in performance follows to about the age of 70 years, when the rate of decline increases. However, the chronic effects of training are maintained for a long time, making older athletes a representative model for studying the ageing process in highly physically active individuals $[2,10,12$, $15,18,21,22]$.

Functional capacity can be classically divided into anaerobic and aerobic components, although they always overlap with varying predomination of one, depending on the type and duration of physical activity. Parameters of anaerobic capacity are not measured and reported that often, and are unfairly neglected throughout literature, especially in studies with inactive and ageing populations $[5,6,20]$. However, these parameters are also very useful in providing deep insight into persons' functional status and adaptation mechanisms during growth and ageing. Several mechanisms were reported to be responsible for the decline in anaerobic power and capacity with ageing. Although the onset of anaerobic power and capacity decline is still a matter of debate [4], most quantitative and qualitative changes which contribute to its reduction are reported in the literature. Skeletal muscle mass loss [9, 14], decreased energy substrate utilization and contractile properties modifications [9], as well as neuromuscular detrimental changes [1] lead to lower anaerobic capacity in older active and non-active populations. If these contributing factors show different kinetics, they may affect anaerobic parameters of muscle power and capacity in different phases of the ageing process.

One of the most popular tests used for anaerobic capacity assessment is the Wingate anaerobic test [3]. The Wingate test, although simple, very popular and widely used for anaerobic power and capacity assessment, because it is objective, valid, reliable, reproducible and sensitive enough to register even smaller changes in anaerobic capability, is an all-out test and can produce substantial discomfort in the participant, especially in non-active subjects. Because the test has a short but great impact on cardiovascular function, it is not recommended for patients with certain disorders. In addition to WAnT, ergometric measurements on a dynamometer for different muscle groups are also widely used in anaerobic capacity assessment. Testing is comfortable and provides several anaerobic parameters: muscle strength, power, work and contraction velocity

Therefore, the aim of the present study was to present anaerobic functional parameters in master athletes of different ages and to compare their results with younger athletes. Also, the aim was to compare the decline among different strength and power parameters in relation to increasing age and to correlate values obtained by 2 different anaerobic tests, Wingate and ergometry testing.

\section{Materials and Methods}

Participants were informed about the study protocol and gave informed consent prior to the testing.

\section{Participants}

The study was conducted on 60 male athletes aged $30-62$ years engaged in different sports: marathon (9), athletics (2), kayaking

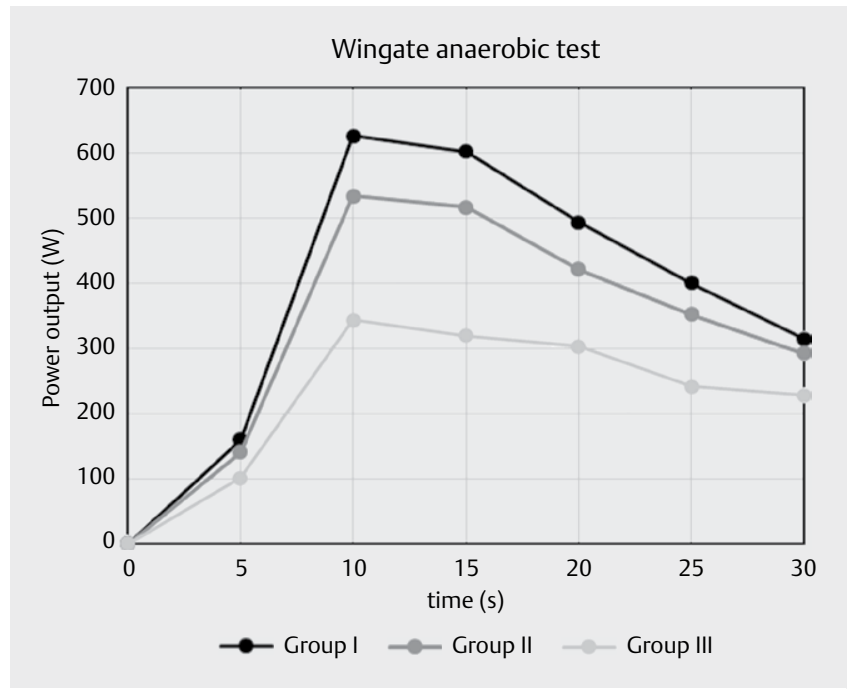

Fig. 1 Values of average anaerobic power in six 5-s intervals during the Wingate anaerobic test in all 3 groups of athletes. Each dot presents the group average anaerobic power value in the calculated 5-s test interval.

(7), mountain climbing (1), cycling (2), football (15), basketball (6), volleyball (2), rowing (1), swimming (1), body building (3), savate boxing (3), boxing (1), judo (2), volleyball (4), and wrestling (1). They were divided into 3 different age groups: group I 30-35 years $\left(n_{1}=35\right.$, age $\left.32.23 \pm 1.5 \mathrm{y}, \mathrm{BW} 87.76 \pm 12.2 \mathrm{~kg}, \mathrm{BH} 186.14 \pm 9.2 \mathrm{~cm}\right)$, group II 35-45 years ( $\mathrm{n}_{2}=15$, age $39.17 \pm 3.6 \mathrm{y}, \mathrm{BW} 86.4 \pm 11.7 \mathrm{~kg}$, $\mathrm{BH} 183.47 \pm 8.6 \mathrm{~cm})$, and group III above 46 years $\left(\mathrm{n}_{3}=10\right.$, age $52.1 \pm 4.5$ y, BW $80.9 \pm 12.7 \mathrm{~kg}, \mathrm{BH} 177.2 \pm 6.6 \mathrm{~cm}$ ). Athletes from the first group, as the control group, were involved at the competition level in their sports, with a training frequency 5-6 times per week, whereas athletes from other 2 groups were competitors with a training frequency of 3-5 times per week. They were all healthy and volunteered for the study. Each participant completed basic anthropometric measurements, and 2 laboratory tests, the Wingate anaerobic test and ergometry measurements for 3 main muscle groups: elbow flexors, elbow extensors, and knee extensors. The study was carried out in accordance with the Declaration of Helsinki and meets the ethical standards of the Journal [7].

\section{Wingate anaerobic test (WAnT)}

After initial warm-up, all participants performed a 30-s all-out test on a bicycle ergometer [16]. Data were recorded and post-processed by software, and the following parameters were displayed: peak power (PP) as maximal power output accomplished during test in watts, explosive power (EP) as PP/time to peak in watts/s, mean power (MP) as average power output throughout the test in watts, work $(\mathrm{W})$ in joules, fatigue index $(\mathrm{FI})$ as percentage decline (\%) in power from PP to $\mathrm{P} 30$ (power output in the $30^{\text {th }}$ second) and power decline (PD) as the average decrease in power from PP to the end of the test in W/s ( $\vee$ Fig. 1).

\section{Ergometry testing}

Muscle strength parameters of elbow flexors and extensors, as well as knee extensors were measured by dynamometry. All ergometry 
measurements were conducted on Concept 2 Dyno isoacceleration dynamometer (Concept 2, Inc. Morrisville, Vermont, USA). Participants were familiarized in detail with the testing procedure. After an initial 10-min warm-up, muscle strength parameters of elbow flexors, elbow extensors, and knee extensors were measured.

For the purpose of the ergometry measurements, participants were in 3 different positions: sitting bench press, leg press, and sitting bench pull position for elbow extensors, knee extensors, and elbow flexors strength measurements, respectively. Before measurements, subjects performed 3 test pre-contractions to introduce them to the performance technique. After 5-min rest periods between different muscle group measurements, participants performed 5 maximal contractions against constant acceleration of the dynamometer. Each contraction lasted for $1 \mathrm{~s}$, with $2 \mathrm{~s}$ of decontraction phase. Values for every contraction were shown on the display of the dynamometer and registered by the examiner. Average and maximal load in $\mathrm{kg}$ (L and Lmax), average (MS) and maximal muscle strength (MSmax) out of 5 maximal contractions, muscle work (W), power $(\mathrm{P})$ and contraction velocity (vel) for each muscle groups were analysed. Average load and muscle strength represented the mean value of 5 individual contractions, whereas maximal load and strength was the best scored result from those 5 contractions.

\section{Statistical analysis}

Data were presented as mean \pm SD. Also, a $95 \%$ confidence interval was calculated for each parameter and presented as an interval from the lower limit (LL) to the upper limit (UL) together with average values in > Table 1, 2, as mean \pm SD (CI LL-CI UL). A 2-tailed t-test for groups with unequal variance was used to express the difference between the control group of athletes and master athletes in 2 older groups. Pearson's correlation coefficient was used to describe the relation between different anaerobic strength parameters, as well as the relation between athletes' age and test results. Statistical significance was set at $p \leq 0.05$, unless otherwise indicated. All analyses were performed using Microsoft Office Excel 2007 software.

\section{Results \\ Anaerobic strength and power parameters}

Anaerobic strength and power parameters measured by WAnT for all 3 groups of participants are presented in > Table 1. It is noticeable that all measured parameters showed significantly lower values in group III when compared with both groups I (for absolute values $\mathrm{p} \leq 0.001$, when parameters were expressed in relation to

- Table 1 Anaerobic power parameters in athletes of different age groups measured by application of the Wingate anaerobic test (all parameters are presented as mean \pm standard deviation, in absolute values and in relation to their body weight, with a $95 \%$ confidence interval (Cl) expressed for each value as the range from the lower to upper limits).

\begin{tabular}{|c|c|c|c|}
\hline WAnT & $\mathbf{I}$ & II & III \\
\hline $\mathrm{PP}(\mathrm{W})$ & $692.94 \pm 216.53$ & $593.40 \pm 185.91$ & $381.50 \pm 117.62^{*}$ \\
\hline $\mathrm{Cl}$ & (621.21-764.68) & (499.32-687.48) & $(308.60-454.40)$ \\
\hline $\mathrm{PP} / \mathrm{BW}(\mathrm{W} / \mathrm{kg})$ & $7.94 \pm 2.30$ & $6.84 \pm 1.92$ & $4.65 \pm 0.80^{*}$ \\
\hline $\mathrm{Cl}$ & $(7.18-8.70)$ & $(5.87-7.81)$ & $4.15-5.15$ \\
\hline $\mathrm{EP}(\mathrm{W} / \mathrm{s})$ & $101.37 \pm 39.25$ & $90.46 \pm 35.66$ & $50.21 \pm 22.72 *$ \\
\hline $\mathrm{Cl}$ & $(88.37-114.37)$ & $(72.41-108.51)$ & $36.13-64.29$ \\
\hline $\mathrm{EP} / \mathrm{BW}(\mathrm{W} / \mathrm{s} / \mathrm{kg})$ & $1.16 \pm 0.41$ & $1.04 \pm 0.39$ & $0.60 \pm 0.20^{*}$ \\
\hline $\mathrm{Cl}$ & $(1.02-1.29)$ & $(0.85-1.24)$ & $(0.48-0.73)$ \\
\hline $\mathrm{MP}(\mathrm{W})$ & $440.60 \pm 117.44$ & $385.91 \pm 107.55$ & $266.68 \pm 65.53^{*}$ \\
\hline $\mathrm{Cl}$ & $(401.69-479.50)$ & $(331.49-440.34)$ & $(226.07-307.30)$ \\
\hline MP/BW (W/kg) & $5.06 \pm 1.26$ & $4.47 \pm 1.19$ & $3.27 \pm 0.44^{*}$ \\
\hline $\mathrm{Cl}$ & $(4.64-5.47)$ & $(3.87-5.08)$ & $(3.00-3.54)$ \\
\hline$W(\jmath)$ & $13217.89 \pm 3523.06$ & $11577.33 \pm 3226.42$ & $8000.50 \pm 1965.85 *$ \\
\hline $\mathrm{Cl}$ & $(12050.71-14385.06)$ & $(9944.57-13210.10)$ & (6782.08-9218.92) \\
\hline W/BW (J/kg) & $151.69 \pm 37.84$ & $134.25 \pm 35.77$ & $98.19 \pm 13.15^{*}$ \\
\hline $\mathrm{Cl}$ & $(139.16-164.23)$ & (116.15-152.35) & (90.04-106.34) \\
\hline $\mathrm{Fl}(\%)$ & $49.05 \pm 12.89$ & $45.78 \pm 10.77$ & $34.62 \pm 11.44^{*}$ \\
\hline $\mathrm{Cl}$ & $(44.78-53.32)$ & $(40.33-51.23)$ & $(27.53-41.71)$ \\
\hline $\mathrm{FI} / \mathrm{BW}(\% / \mathrm{kg})$ & $0.57 \pm 0.16$ & $0.53 \pm 0.11$ & $0.42 \pm 0.09^{*}$ \\
\hline $\mathrm{Cl}$ & $(0.52-0.62)$ & $(0.47-0.59)$ & $(0.37-0.48)$ \\
\hline PD (W) & $20.01 \pm 7.24$ & $16.29 \pm 6.60$ & $8.95 \pm 4.84^{*}$ \\
\hline $\mathrm{Cl}$ & $(17.61-22.41)$ & $(12.94-19.63)$ & (5.95-11.95) \\
\hline PD/BW (W/kg) & $0.23 \pm 0.08$ & $0.19 \pm 0.06$ & $0.11 \pm 0.04^{*}$ \\
\hline $\mathrm{Cl}$ & $(0.20-0.26)$ & $(0.15-0.22)$ & $(0.08-0.13)$ \\
\hline \multicolumn{4}{|c|}{ PP - peak power, EP - explosive power, MP - mean power, W - work, FI - fatigue index, PD - power decline, BW - body weight } \\
\hline
\end{tabular}


- Table 2 Values of measured ergometric parameters for tested elbow flexor, elbow extensor and knee extensor muscles in 3 groups of participants (all parameters are presented as mean \pm standard deviation, with a $95 \%$ confidence interval $(\mathrm{Cl})$ expressed for each value as the range from the lower to upper limits).

\begin{tabular}{|c|c|c|c|c|}
\hline & D & 1 & II & III \\
\hline \multirow[t]{14}{*}{$\mathrm{EF}$} & $\mathrm{L}(\mathrm{kg})$ & $80.74 \pm 18.97$ & $81.40 \pm 19.37$ & $67.60 \pm 16.59$ \\
\hline & $\mathrm{Cl}$ & $(74.46-87.03)$ & $(71.60-91.20)$ & (57.31-77.89) \\
\hline & $\operatorname{Lmax}(\mathrm{kg})$ & $87.37 \pm 19.80$ & $87.80 \pm 20.21$ & $73.10 \pm 18.54$ \\
\hline & $\mathrm{Cl}$ & $(80.81-93.93)$ & $(77.57-98.03)$ & $(61.61-84.59)$ \\
\hline & MS (N) & $792.09 \pm 186.11$ & $798.53 \pm 190.03$ & $663.16 \pm 162.79$ \\
\hline & $\mathrm{Cl}$ & $(730.43-853.74)$ & $(702.36-894.70)$ & $(562.26-764.05)$ \\
\hline & $\operatorname{MSmax}(\mathrm{N})$ & $857.11 \pm 194.21$ & $861.32 \pm 198.23$ & $717.11 \pm 181.92$ \\
\hline & $\mathrm{Cl}$ & $(792.77-921.46)$ & $(761.00-961.63)$ & $(604.36-829.86)$ \\
\hline & $P(W)$ & $371.86 \pm 124.00$ & $375.80 \pm 121.56$ & $273.70 \pm 97.32 * *$ \\
\hline & $\mathrm{Cl}$ & $(330.78-412.94)$ & $(314.28-437.32)$ & $(213.38-334.02)$ \\
\hline & $\mathrm{W}(\mathrm{J})$ & $439.97 \pm 120.22$ & $433.73 \pm 118.11$ & $340.10 \pm 83.83^{* *}$ \\
\hline & $\mathrm{Cl}$ & $(400.14-479.80)$ & $(373.96-493.50)$ & (288.14-392.06) \\
\hline & Vel $(\mathrm{mm} / \mathrm{s})$ & $465.03 \pm 47.86$ & $470.53 \pm 59.92$ & $405.90 \pm 44.94^{* *}$ \\
\hline & $\mathrm{Cl}$ & $(449.17-480.88)$ & $(440.21-500.86)$ & $(378.05-433.75)$ \\
\hline \multirow[t]{14}{*}{$\mathrm{EE}$} & $\mathrm{L}(\mathrm{kg})$ & $80.14 \pm 14.72$ & $78.60 \pm 20.37$ & $64.90 \pm 19.05$ \\
\hline & $\mathrm{Cl}$ & $(75.27-85.02)$ & $(68.29-88.91)$ & $(53.09-76.71)$ \\
\hline & $\operatorname{Lmax}(\mathrm{kg})$ & $84.26 \pm 15.27$ & $82.93 \pm 20.86$ & $68.70 \pm 19.29$ \\
\hline & $\mathrm{Cl}$ & $(79.20-89.31)$ & $(72.38-93.49)$ & $(56.75-80.65)$ \\
\hline & MS (N) & $786.20 \pm 144.43$ & $771.07 \pm 199.87$ & $636.67 \pm 186.90$ \\
\hline & $\mathrm{Cl}$ & $(738.35-834.05)$ & $(669.92-872.21)$ & $(520.83-752.51)$ \\
\hline & $\operatorname{MSmax}(\mathrm{N})$ & $826.56 \pm 149.77$ & $813.58 \pm 204.62$ & $673.95 \pm 189.21$ \\
\hline & $\mathrm{Cl}$ & $(776.95-876.18)$ & $(710.03-917.13)$ & $(556.67-791.22)$ \\
\hline & $P(W)$ & $341.17 \pm 96.10$ & $323.07 \pm 102.45$ & $239.60 \pm 93.55^{* *}$ \\
\hline & $\mathrm{Cl}$ & $(309.34-373.01)$ & $(271.22-374.91)$ & $(181.62-297.58)$ \\
\hline & $\mathrm{W}(\mathrm{J})$ & $377.09 \pm 73.67$ & $370.07 \pm 107.77$ & $281.50 \pm 67.84^{* *}$ \\
\hline & $\mathrm{Cl}$ & $(352.68-401.49)$ & $(315.53-424.60)$ & $(239.45-323.55)$ \\
\hline & Vel $(\mathrm{mm} / \mathrm{s})$ & $439.97 \pm 54.39$ & $424.60 \pm 55.10$ & $377.80 \pm 40.79 * *$ \\
\hline & $\mathrm{Cl}$ & (421.95-457.99) & $(396.72-452.48)$ & $(352.52-403.08)$ \\
\hline \multirow[t]{14}{*}{ KE } & $\mathrm{L}(\mathrm{kg})$ & $170.71 \pm 20.84$ & $151.53 \pm 36.69^{*}$ & $131.20 \pm 36.69$ \\
\hline & $\mathrm{Cl}$ & $(163.81-177.62)$ & $(132.97-170.10)$ & $(108.46-153.94)$ \\
\hline & $\operatorname{Lmax}(\mathrm{kg})$ & $182.69 \pm 20.95$ & $161.60 \pm 36.50^{*}$ & $141.10 \pm 36.16$ \\
\hline & $\mathrm{Cl}$ & $(175.75-189.63)$ & $(143.13-180.07)$ & $(118.69-163.51)$ \\
\hline & MS (N) & $1674.71 \pm 204.49$ & $1486.54 \pm 359.94^{*}$ & $1287.07 \pm 359.90$ \\
\hline & $\mathrm{Cl}$ & $(1606.96-1742.45)$ & $(1304.39-1668.70)$ & $(1064.01-1510.14)$ \\
\hline & $\operatorname{MSmax}(\mathrm{N})$ & $1792.15 \pm 205.48$ & $1585.30 \pm 358.03^{*}$ & $1384.19 \pm 354.68$ \\
\hline & $\mathrm{Cl}$ & $(1724.07-1860.22)$ & $(1404.11-1766.48)$ & $(1164.36-1604.02)$ \\
\hline & $\mathrm{P}(\mathrm{W})$ & $868.77 \pm 206.03$ & $757.20 \pm 224.08$ & $587.70 \pm 219.93$ \\
\hline & $\mathrm{Cl}$ & $(800.51-937.03)$ & $(643.80-870.60)$ & $(451.39-724.01)$ \\
\hline & $\mathrm{W}(\mathrm{J})$ & $632.54 \pm 118.58$ & $579.60 \pm 157.20$ & $462.10 \pm 136.23$ \\
\hline & $\mathrm{Cl}$ & (593.26-671.83) & (500.05-659.15) & $(377.66-546.54)$ \\
\hline & Vel $(\mathrm{mm} / \mathrm{s})$ & $557.34 \pm 63.72$ & $531.27 \pm 72.72$ & $486.40 \pm 79.80$ \\
\hline & $\mathrm{Cl}$ & $(536.23-578.45)$ & $(494.47-568.07)$ & $(436.94-535.86)$ \\
\hline \multicolumn{5}{|c|}{$\begin{array}{l}\text { EF - elbow flexion, EE - elbow extension, KE - knee extension, L - load, Lmax - maximal load, MS - muscle strength, MSmax - maximal muscle } \\
\text { strength, } \mathrm{P} \text { - power, } \mathrm{W} \text { - work, Vel - contraction velocity }\end{array}$} \\
\hline \multicolumn{5}{|c|}{${ }^{*}$ statistically significant difference, when comparing group II with group I $(p \leq 0.05)$} \\
\hline \multicolumn{5}{|c|}{${ }^{*}{ }^{*}$ statistically significant difference, when comparing group III with group II $(\mathrm{p} \leq 0.05)$} \\
\hline
\end{tabular}


- Table 3 Percentage decline in ergometric parameters in both groups of older athletes when compared with control group (set as 100\%).

\begin{tabular}{|c|r|r|r|r|r|}
\hline & \multicolumn{1}{c|}{ MS } & MSmax & \multicolumn{1}{c|}{$\mathbf{P}$} & \multicolumn{1}{c|}{ W } & \multicolumn{1}{c|}{ Vel } \\
\hline EF II & +0.8 & +0.5 & +1 & -1.4 & +1 \\
\hline EF III & -16.3 & -16.3 & -26.4 & -22.7 & -12.9 \\
\hline EE II & -1.9 & -1.6 & -5.3 & -1.9 & -3.5 \\
\hline EE III & -19.0 & -18.5 & -29.8 & -25.3 & -14.1 \\
\hline KE II & -11.2 & -11.5 & -12.8 & -8.4 & -4.7 \\
\hline KE III & -23.1 & -22.8 & -32.3 & -26.9 & -12.7 \\
\hline
\end{tabular}

body weight $p \leq 0.01$ ) and II (for both absolute values and when expressed in relation to body weight $\mathrm{p} \leq 0.05$ ). However, when group I was compared with group II, there were no significant differences registered for any of measured parameters (for PP, EP, MP, W, FI, and PD $p>0.05$ ). The same difference was present when parameters were expressed in relation to body weight (PP/BW, EP/BW, MP/ $\mathrm{BW}, \mathrm{W} / \mathrm{BW}, \mathrm{FI} / \mathrm{BW}, \mathrm{PD} / \mathrm{BW}, \mathrm{p}>0.05)$. Additional analysis in groups I and II for comparison between predominantly anaerobic and aerobic sports was performed and showed no significant differences in WAnT parameters between these 2 subgroups.

Ergometric parameters for 3 muscle groups (elbow flexors, elbow extensors and knee extensors) in our participants are presented in $>$ Table 2 . Group III had significantly lower values for all ergometric parameters when compared with group I (for EF $p \leq 0.05$, for $E E$ and $K E p \leq 0.01$ ). When group III was compared to group II, significantly lower values in older athletes were registered for parameters of elbow flexor and extensor power, work and contraction velocity $(p \leq 0.05)$. Since some athletes were engaged in sports with predominant use of upper or lower extremities, additional analysis was performed in subgroups of athletes with predominant use of the lower limbs, in order to test differences between arm muscles without additional training effect. In a total of 29 athletes, significant differences were observed in elbow flexion power and velocity between groups II and III, and for all arm parameters between groups I and III (except for work of elbow flexors). Different distribution of arm strength differences between subgroups of athletes with predominant leg contractions implies that ageing may affect elbow flexors and extensors differently, or that ageing can have a different effect on predominantly trained and untrained muscle groups.

In knee extensors, no significant difference was found between groups II and III ( $p>0.05)$. Group II had significantly lower values for parameters of knee extensor load, strength, maximal load and strength when compared with group I $(p \leq 0.05)$. Lower values were registered in older athletes with different dynamics of decline among parameters. The percentage decline in all ergometric parameters is presented in $>$ Table $\mathbf{3}$.

\section{Correlations}

A significant correlation was registered when comparing WAnT parameters of PP and EP with ergometric parameters for knee extensors (KE) in all 3 groups of participants (for PP/KE power: $r$ was 0.59 in group I, 0.85 in group II, and 0.90 in group III; for EP/KE power: $r$ was 0.51 in group I, 0.8 in group II, and 0.71 in group III, $p \leq 0.05$ ).

A strong positive correlation was observed between different arm flexor and extensor ergometry parameters in the same age group of participants ( $r$ from 0.74 to 0.97 ). When sample size was included, the significance level for all $r$ values was $p \leq 0.001$, except for the work parameter in group II and the contraction velocity parameter in group III, where $r$ was 0.74 and 0.83 , respectively $(\mathrm{p} \leq 0.01)$.

When testing the possible influence of athlete's age on maximal anaerobic power results (PP), a significant but moderate negative correlation was registered only in group III (the oldest participants) with $r=-0.40(p \leq 0.05)$, whereas in group I and II $r$ was -0.19 and -0.26 , respectively $(p>0.05)$.

\section{Discussion}

Ageing is a process that can be considered inherent, universal, progressive and decremental [19]. Physical activity presents one of the most important evolutionary factors that have changed in our modern environment and undoubtedly lack of it is considered significant in the development of various disorders during a lifespan, especially during growth and ageing.

In the present study, different parameters of anaerobic strength and power were tested in older athletes of different ages by using 2 popular anaerobic tests, the Wingate and ergometry. Decline in parameters of anaerobic strength and power, although not harmonic, was prominent in fifth and sixth decade, whereas master athletes aged 35-45 years showed variable decline in anaerobic strength and power in comparison to younger athletes. Because some athletes in groups I and II were engaged in anaerobic types of sports, an additional comparison between anaerobic and aerobic sports was performed. No difference in peak anaerobic power between those subgroups can be explained by the fact that these athletes have had predominately aerobic type of training after the age of 30 years.

All Wingate anaerobic test parameters were significantly lower in the group of athletes of 46 years and older, whereas in the group of athletes aged 35-45 years no significant difference was registered when compared with younger ones. The same distribution in significance was present when parameters were expressed in relation to body weight, therefore the registered decline cannot be explained solely by the loss of muscle mass in the fifth and sixth decade of life. Grassi et al. reported that the peak anaerobic power decline at age 75 years is about $50 \%$ of the value measured at age 20 years (corresponding to a reduction of about $1 \% /$ year), and that up to age 45 such deterioration was mainly attributable to qualitative factors, whereas after that age quantitative factors (loss in muscle mass) were also involved [6]. Also, Chamari et al. showed $42.7 \%$ lower peak anaerobic power values in older athletes (65.1 $y)$ in comparison with younger ones ( $24.8 \mathrm{y}$ ) with mean values of $624 \mathrm{~W}$ compared with $1089 \mathrm{~W}$ [5]. The present study showed $14.4 \%$ and $44.9 \%$ lower values of PP in older athletes aged 35-45 years and $46-62$ years respectively, when compared with younger ones ( $30-35$ years).

However, it would be essential to introduce regional muscle mass values when expressing anaerobic strength parameters in older individuals through parameter of muscle quality (strength or power/muscle mass), especially after sixth decade, as physiological muscle mass loss counts for strength decline and decrease in 
anaerobic power. Muscle quality could give more precise insight into a participant's functional capacity and strength increase as a result of different training protocols, and would also allow better comparison of strength decline between active and non-active older populations, as well as in their comparison to younger athletes of different sports [5, 6, 17].

When discussing potential factors that influence anaerobic power decline with age, energy substrate utilisation pathways are of great importance. The amount of available creatine phosphate and adenosine triphosphate (ATP) in muscle are responsible for fast energy production at the beginning of exercise. While lactate concentrations were measured in master athletes [13], no data are available so far on master athletes' phosphocreatine and ATP muscle concentrations, although data were reported in a non-athletic population [8]. Application of non-invasive imaging procedures like magnetic resonance spectroscopy could add to the existing knowledge on kinetics of high-energy phosphates concentrations in ageing muscles of physically active individuals.

When analysing ergometric parameters, a more prominent decline in some parameters for specific muscles in group II can be partially attributed to different sport activities and different training regimes. In group III, all measured parameters had the lowest values compared with younger athletes in group I. When group II and III were compared, significantly lower values of ergometric parameters were registered for power, work and velocity of elbow flexors and extensors, but not for load and strength values. Additional statistical analysis on arm parameters was performed with 3 subgroups of athletes engaged in sports with predominant leg contractions. Significant differences were observed in elbow flexion power and velocity between groups II and III, and for all arm parameters between groups I and III (except for work of elbow flexors). The different distribution of arm strength differences between subgroups of athletes with predominant leg contractions implies that ageing may affect elbow flexors and extensors differently, or that ageing can have a different effect on predominantly trained and untrained muscle groups. However, this sample is small for analysing this impact with enough statistical power, and upper arm data is discussed only in correlation analyses of different muscle groups in the same participant. Analysis of different upper arm muscle group strength parameters for the subgroups of athletes with predominant use of lower limbs in their sports shows mainly an ageing effect on these parameters, but no training influence on upper arm muscles. Deeper analysis of differences in ageing vs. training influence on upper and lower muscles strength could not be performed without data on muscle size.

For knee extensors, no significant difference for any parameter was registered between group II and III. Similarly, Swank et al. reported no difference in knee extensor strength between athletes aged $40-59$ years and over 60 years and their age-matched, moderately active controls. They explained that the lack of significant differences in knee extensor strength and flexibility between groups may relate to the joints measured and previous injuries of tennis players [20].

When comparing different ergometric parameters and different muscle groups, this study confirmed that different strength parameters "age" in a different fashion. Very low and equal decline in contraction velocity parameters was registered for all muscle groups in older participants when compared with younger ones, as emphasized in > Table 3. It points to the possibility of longer preservation of some muscle parameters like velocity through the ageing process, and it is still unknown whether it is related solely to previous levels of physical activity, like in master athletes, or has a more complex explanation. Maybe neuromuscular adaptation, as a part of the skeletal muscle adaptation mechanism to increased load, could be maintained as a result of chronic adaptation to training, and can increase coordination and activation of motor units during contraction when muscle mass is lost, and perhaps also slow decreased mobility in later life. This finding could be used in designing training protocols for older participants by introducing strength training more often. It remains questionable as to whether previously inactive individuals can be easily trained, how much training might improve coordination for accomplishing everyday activities, and would training eventually decrease disability in ageing. The study by Chamari et al. also showed that the age-related difference in force was significantly greater than that in contraction velocity, with the velocity/force coefficient $18.4 \%$ higher in older athletes, with an approximately $4.4 \%$ increase in difference per decade [5]. The findings confirm in humans the investigations in animals showing that strength is more influenced than velocity by the muscle alterations with increasing age [11].

The present study showed a significant correlation between parameters of strength measured by 2 different tests, the Wingate test and knee extensor ergometry. This is important because the Wingate test is all-out test, and although it is simple to perform and does not require special skills from participants, it could be difficult to apply in older participants or those with certain disorders because it is very challenging for the cardiovascular and musculoskeletal systems. Therefore, some parameters of anaerobic strength and power could be reliably obtained by ergometry, which is much easier for participants to perform. Knee extensor ergometry in this study was performed as a 15-s test with 5 consecutive maximal knee extensions against resistance, each lasting $1 \mathrm{~s}$, and with $2 \mathrm{~s}$ knee flexion without resistance after each extension.

Non-significant correlation between age and maximal anaerobic power parameters in younger athletes, as well as existence of moderate negative correlation between those 2 parameters in the oldest group of athletes additionally explains the influence of age on functional capacity and anaerobic power decline in the fifth and sixth decades of life, which is in compliance with the literature data $[5,6,12,21]$.

The present study showed a variety of anaerobic strength and power parameters in athletes of different ages. Master athletes are very interesting and unique population group in which the ageing process can be observed in the light of regular and chronic effects of physical activity. Aerobic training predominates in older individuals, together with aerobic types of regular physical activities. Therefore, the anaerobic component of functional capacity is less studied throughout literature, and similar studies with older participants engaged in different levels of physical activity are needed in the future. Also, the effects of different anaerobic training protocols on the increase in anaerobic functional capacity in older participants can add to the existing knowledge of neuromuscular and metabolic adaptation mechanisms and dynamics of their decline through ageing process. 
Because physical activity is recognized as a part of treatment strategy for numerous disorders, it is important to include the master athlete population in researching the ageing process and its pathophysiological mechanisms.

\section{Conclusions}

In conclusion, master athletes represent a very interesting and significant population for studying the chronic effects of physical activity on functional capacity and its changes during ageing. Anaerobic capacity also provides valuable information on adaptation to physical activity with increasing age and should be studied more closely together with aerobic capacity parameters. In this study, peak anaerobic power was lower for $14.4 \%$ in athletes aged $35-45$ years when compared with those from 30-35 years. In athletes aged $46-62$ years, the decrease in peak power was $44.9 \%$. The ergometry parameter of muscle strength and power showed greater decline with age (up to $32.3 \%$ ) in comparison with contraction velocity decrease (up to $14.1 \%$ ).

\section{Conflict of interest}

Author has declared no conflict of interest.

\section{References}

[1] Aagard P, Suetta C, Caseroti P, Magnusson SP, Kjaer M. Role of the nervous system in sarcopenia and muscle atrophy with aging: strength training as a countermeasure. Scand J Med Sci Sports 2010; 20: 49-64

[2] Akkari A, Machin D, Tanaka H. Greater progression of athletic performance in older Masters athletes. Age Ageing 2015; DOI:10.1093/ageing/afv023

[3] Bar-Or O. The Wingate anaerobic test. An update on methodology, reliability and validity. Sports Med 1987; 4: 381-394

[4] Capelli C, Rittweger J, Bruseghini P, Calabria E, Tam E. Maximal aerobic power and anaerobic capacity in cycling across the age spectrum in male master athletes. Eur J Appl Physiol 2016; DOI:10.1007| s00421-016-3396-9

[5] Chamari K, Ahmaidi S, Fabre C, Masse-Biron J, Prefaut CH. Anaerobic and aerobic peak power output and the force-velocity relationship in endurance-trained athletes: effects of aging. Eur J Appl Physiol 1995; 71: $230-234$
[6] Grassi B, Cerretelli P, Narici MV, Marconi C. Peak anaerobic power in master athletes. Eur J Appl Physiol 1991; 62: 394-399

[7] Harriss DJ, Atkinson G. Ethical standards in sport and exercise science research: 2016 update. Int J Sports Med 2015; 36: 1121-1124

[8] Kerksich CM, Roberts MD, Dalbo V], Sunderland KL. Intramuscular phosphagen status and the relationship to muscle performance across the age spectrum. Eur J Appl Physiol 2016; 116: 115-127; DOI: 10.1007/s00421-015-3246-1

[9] Korhonen MT, Cristea A, Alen M, Hakkinen K, Sipila S, Mero A, Viitasalo JT, Larsson L, Suominen H. Aging, muscle fiber type, and contractile function in sprint-trained athletes. J Appl Physiol 2006; 101: 906-917

[10] Kusy K, Zielinski J. Ageing, aerobic capacity and insulin sensitivity in masters athletes: endurance and speed-power training benefits. Trends Sport Sci 2014; 2: 73-84

[11] Larsson L, Grimby G, Karlsson J. Muscle strength and speed of movement in relation to age and muscle morphology. J Appl Physiol Respir Environ Exerc Physiol 1979; 46: 451-456

[12] Lazarus NR, Harridge SDR. Inherent ageing in humans: the case for studying master athletes. Scand J Med Sci Sports 2007; 17: 461-463

[13] Marsh GD, Paterson DH, Govindasamy D, Cunningham DA. Anaerobic power of the arms and legs of young and older men. Exp Physiol 1999; 84: 589-579

[14] Narici MV, Maffulli N. Sarcopenia: characteristics, mechanisms and functional significance. Br Med Bull 2010; 95: 139-159

[15] Peiffer J, Abbiss CR, Sultana F, Bernard T, Brisswalter J. Comparison of the influence of age on cycling efficiency and the energy cost of running in well-trained triathletes. Eur J Appl Physiol 2016; 116: 195-201

[16] Popadic Gacesa J, Barak O, Grujic N. Maximal anaerobic power test in athletes of different sport disciplines. J Strength Condit Res 2009; 23: 751-755

[17] Popadic Gacesa JZ, Nedeljkovic A, Ponorac N, Krulanovic R, Grujic NG. Assessment of elbow extensors endurance in young athletes. Int J Sports Med 2012; 33: 1005-1009

[18] Rittweger J, di Prampero PE, Maffulli N, Narici MV. Sprint and endurance power and ageing: an analysis of master athletic world records. Proc R Soc 2009; 276: 683-689

[19] Strehler BL. Time, Cells, and Aging. New York: Academic Press; 1962

[20] Swank AM, Condra S, Yates JW. Effect of long term tennis participation on aerobic power, body composition, muscular strength flexibility and serum lipids. Sports Med Training Rehab 1998; 8: 99-112

[21] Tanaka H, Seals DR. Invited Review: Dynamic exercise performance in Masters athletes: insight into the effects of primary human aging on physiological functional capacity. J Appl Physiol 2003; 95: 2152-2162

[22] Tanaka H, Seals DR. Endurance exercise performance in Master athletes: age-associated changes and underlying physiological mechanisms. J Physiol 2008; 586: 55-63 